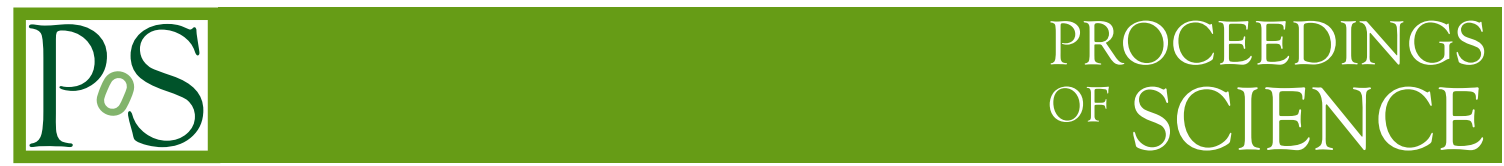

\title{
Search for exotics long-lived particles with ATLAS at the LHC
}

\author{
Antonio POLICICCHIO*i \\ INFN Cosenza, Cosenza, Italy \\ E-mail: antonio.policicchiodcern.ch
}

Data taken in 2011 with the ATLAS detector at the Large Hadron Collider have been used to search for physics beyond the Standard Model. Results are presented based on a luminosity of $2 \mathrm{fb}^{-1}$ of $\sqrt{(s)}=7 \mathrm{TeV}$ proton-proton collisions focusing on final states with exotic long-lived neutral particles. No evidence of new physics is found.

The European Physical Society Conference on High Energy Physics

18-24 July, 2013

Stockholm, Sweden

\footnotetext{
${ }^{*}$ Speaker.

${ }^{\dagger}$ member of the ATLAS Collaboration
} 


\section{Introduction}

The Standard Model (SM) of particle physics has proven extremely successful, but despite its many successes still remains incomplete. New particles with decay path comparable to the LHC detector dimensions (Long-Lived Particles, LLP) are predicted in many models of physics beyond the SM, such as Hidden Sector scenarios or Supersymmetry (SUSY) models. These particles may be neutral and decay into SM and/or weakly interacting particles that escape the detection. This note presents the searches with ATLAS detector [1] for exotics LLP based on $p p$ collision data collected during 2011 at $\sqrt{s}=7 \mathrm{TeV}$.

\subsection{Hidden sector neutral particles}

Decays far from the interaction point are signatures of long-lived neutral particles. ATLAS has performed a search based on $1.9 \mathrm{fb}^{-1}$ for pairs of back-to-back particles decaying to heavy fermions in the muon system [2]. Such signatures may be due to Higgs decays to pairs of longlived neutral particles $h \rightarrow \pi_{v} \pi_{v}$, where $\pi_{v}$ is a pseudoscalar from a Hidden Sector weakly coupled to the Standard Model sector. Limits have been set as a function of the proper decay length of the $\pi_{v}$ excluding the range $0.5-23 \mathrm{~m}$ depending on the Higgs mass $(120-140 \mathrm{GeV})$ and on the $\pi_{v}$ mass $(20-40 \mathrm{GeV})$. Exclusion limits are shown in Figure 1.

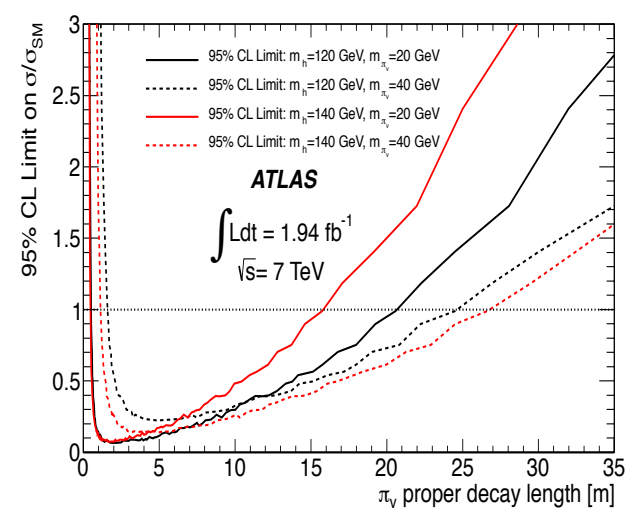

Figure 1: Observed 95\% upper limits on the production cross section for $h \rightarrow \pi_{v} \pi_{v}$ versus the $\pi_{v}$ proper decay length, expressed as a multiple of the SM cross section for Higgs production. Exclusion limits assume $100 \%$ branching ratio for the Higgs decaying to $\pi_{v}$ 's.

A specific model of a neutral long-lived, spinless, exotic particle which has a nonzero branching fraction to dileptons has been used in the search for displaced clusters of leptons (leptonjets). This scenario predicts up to two displaced dilepton vertices in the tracking volume per event. In the study, the model consists of a Higgs boson decaying to new hidden sector particles which finally produce two sets of collimated muon pairs: $h \rightarrow f_{d_{1}}+f_{d_{1}}, f_{d_{1}} \rightarrow f_{d_{2}}+\gamma_{d}, \gamma_{d} \rightarrow l^{+} l^{-}$where $f_{d_{1}}$ is a fermion from the hidden sector, $f_{d_{2}}$ is a stable fermion from the hidden sector, and $\gamma_{d}$ is a dark photon decaying to pairs of leptons. In $1.9 \mathrm{fb}^{-1}$ of data [3] no events consistent with this Higgs boson decay mode are observed. 95\% CL upper limits on the cross section times branching ratio $(\sigma \times B R)$ has been evaluated for the process $H \rightarrow \gamma_{d} \gamma_{d}+X$ ( $X$ refers to the two stable $f_{d_{2}}$ fermions) with the $\gamma_{d}$ mass set to $0.4 \mathrm{GeV}$ (the branching ratio to muons is maximum for this mass value). 
The $(\sigma \times B R)$ is given as a function of the $\gamma_{d}$ mean lifetime, expressed as $c \tau$ for a Higgs mass of 100 and $140 \mathrm{GeV}$. These limits are shown on Figure 2. Table 1 shows the ranges in which the $\gamma_{d} c \tau$ is excluded at the $95 \% \mathrm{CL}$ for $H \rightarrow \gamma_{d} \gamma_{d}+X$ branching ratios of $100 \%$ and $10 \%$.

\begin{tabular}{|c|cc|}
\hline $\begin{array}{c}\text { Higgs boson mass } \\
{[\mathrm{GeV}]}\end{array}$ & $\begin{array}{c}\text { excluded } \mathrm{c} \tau[\mathrm{mm}] \\
\mathrm{BR}(100 \%)\end{array}$ & $\begin{array}{c}\text { excluded } \mathrm{c} \tau[\mathrm{mm}] \\
\mathrm{BR}(10 \%)\end{array}$ \\
\hline 100 & $1 \leq \mathrm{c} \tau \leq 670$ & $5 \leq \mathrm{c} \tau \leq 159$ \\
\hline 140 & $1 \leq \mathrm{c} \tau \leq 430$ & $7 \leq \mathrm{c} \tau \leq 82$ \\
\hline
\end{tabular}

Table 1: Ranges in which $\gamma_{d} c \tau$ is excluded at $95 \%$ CL for 100 and $140 \mathrm{GeV}$ Higgs masses, assuming $100 \%$ and $10 \%$ branching ratio of $H \rightarrow \gamma_{d} \gamma_{d}+X$.
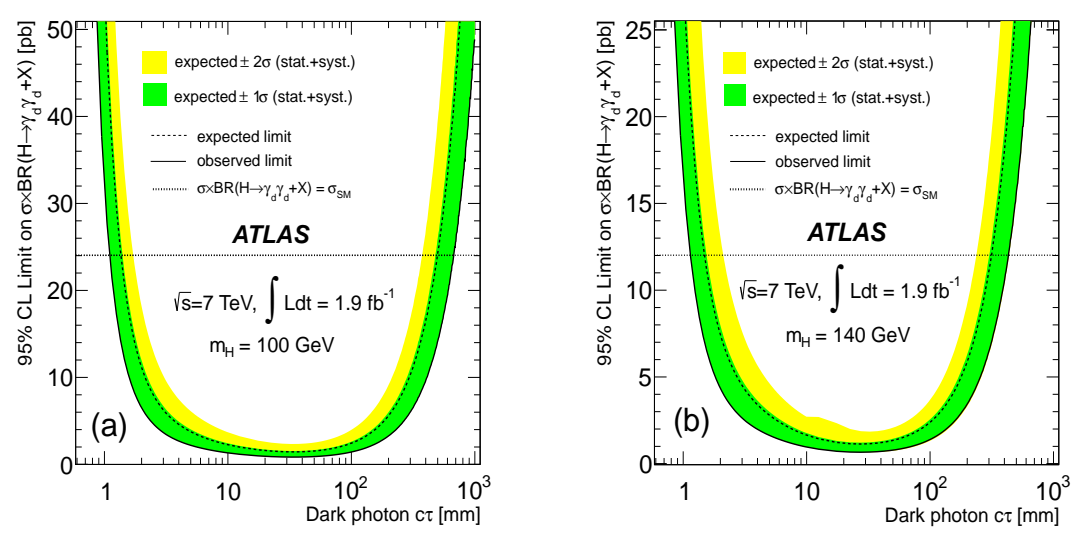

Figure 2: The 95\% upper limits on the $\sigma \times B R$ for the process $H \rightarrow \gamma_{d} \gamma_{d}+X$ as a function of the dark photon $c \tau$ for $\mathrm{m}_{H}=100 \mathrm{GeV}$ and with $\mathrm{m}_{H}=140 \mathrm{GeV}$. The expected limit is shown as the dashed curve and the solid curve shows the observed limit. The horizontal lines correspond to the Higgs boson SM cross sections at the two mass values.

\section{Conclusions}

The search for new phenomena beyond the Standard Model is a very active field at the ATLAS experiment. Recent results for exotic Long-Lived Neutral Particle searches at ATLAS are presented in this note. No excess over the Standard Model expectation has been observed and limits are placed on the various models. The reader is invited to read the detailed papers on each of these analyses.

\section{References}

[1] The ATLAS Collaboration, The ATLAS Experiment at the CERN Large Hadron Collider, JINST 3 (2008) S08003

[2] The ATLAS Collaboration, Search for a light Higgs boson decaying to long-lived weakly-interacting particles in proton-proton collisions at $\sqrt{s}=7$ TeV with the ATLAS detector, Phys. Rev. Lett. 108 (2012) 251801

[3] The ATLAS Collaboration, Search for displaced muonic lepton jets from light Higgs boson decay in proton-proton collisions at $\sqrt{(s)}=7 \mathrm{TeV}$ with the ATLAS detector, Phys. Lett. B 721 (2013) 32 